\title{
APRENDIZAJE POR COMPETENCIAS A TRAVÉS DE LA LITERATURA EN EL GRADO DE EDUCACIÓN INFANTIL: EL TALLER DE POESÍA
}

\author{
Recepción: 24/10/2017 | Revisión: 21/11/2017 | Aceptación: 20/12/2017
}

\section{Noelia MARTÍNEZ HERVÁS}

Universidad Católica de Valencia San Vicente Mártir noelia.martinez@ucv.es

\section{Carme AGUSTÍ APARISI}

Universidad Católica de Valencia San Vicente Mártir carme.agusti@ucv.es

Resumen: El trabajo ${ }^{1}$ que presentamos a continuación describe una experiencia educativa innovadora llevada a cabo en un aula universitaria, concretamente en la asignatura Recursos para la Educación Literaria de cuarto curso de Grado de Educación Infantil de la Facultad de Magisterio de la Universidad Católica de Valencia San Vicente Mártir. La innovación ha consistido en trabajar la poesía mediante la metodología de rincones para después ponerlos en práctica con alumnado de Infantil.

Palabras clave: poesía; subgéneros poéticos; rincones; aprendizaje autónomo; aprendizaje significativo.

COMPETENCY-BASED LEARNING THROUGH LITERATURE IN THE FACULTY OF EDUCATION: THE POETRY WORKSHOP

Abstract: This work describes an innovative experience developed with fourth year students of Primary Education at the Universidad Católica de Valencia San Vicente Mártir through the Resources for Literary Education subject of the Early Years Education degree. The innovation has consisted in working on poetry with a methodology called 'Corners' so the pre-service teachers could put it into practice with Pre-school Education students.

Keywords: poetry; poetic minor genres; corners; autonomous learning; significant learning.

\section{APRENENTATGE PER COMPETĖNCIES A TRAVÉS DE LA LITERATURA EN EL GRAU D'EDUCACIÓ INFANTIL: EL TALLER DE POESIA}

Resum: El treball que presentem descriu una experiència educativa innovadora duta a terme en una aula universitària, concretament en l'assignatura Recursos per a l'Educació Literària de quart curs del Grau d'Educació Infantil de la Facultat de Magisteri de la Universidad Católica de Valencia San Vicente Mártir. La innovació ha consistit a treballar la poesia mitjançant la metodologia dels racons per després posar-los en pràctica amb estudiants d'Infantil.

Paraules clau: poesia; subgèneres poètics; racons; aprenentatge autònom; aprenentatge significatiu.

1 Este estudio se incluye en el marco del grupo de investigación Humanidades Digitales de la Facultad de Magisterio y Ciencias de la Educación y en el grupo de investigación La literatura como recurso para la adquisición de competencias en el Grado de Magisterio. Ambos grupos pertenecen a la Universidad Católica de Valencia San Vicente Mártir. Además se incluye en el marco del proyecto I+D+I MEHHRLYN “Magia, épica e historiografía hispánicas. Relaciones literarias y nomológicas”, FFI2015-64050, dirigido por Alberto Montaner (Ministerio de Economía y Competitividad) y en la Red de Excelencia "Universidad, Innovación y Aprendizaje en la Sociedad del Conocimiento", dirigida por Miguel A. Santos Rego (USC) (Código EDU2017-90651-REDT). 


\section{Introducción: La competencia literaria y la importancia de la poesía}

Proponemos con este trabajo aportar una serie de propuestas concretas respecto a la competencia literaria en el aula de infantil, centrándonos en la poesía, dada nuestra práctica docente y nuestra experiencia en el aula de Magisterio. La preparación de maestros con la nueva reforma del sistema de grados basado en la metodología de Bolonia, proporciona una ocasión inestimable para una nueva manera de trabajar. No tiene sentido estar hablando de cambio metodológico, de educación por competencias, de aprendizaje significativo y otros conceptos innovadores, si continuamos utilizando para la formación de nuestro alumnado las mismas prácticas docentes de antaño. Siguiendo a Zabala (2009), esta formación tendrá también que estar basada en competencias, de manera que las formas de enseñanza tendrán que asumir todas las consecuencias de una metodología que corresponda al conocimiento que tenemos sobre cómo se produce el aprendizaje. Por esto, a nivel universitario y respecto a las competencias, hay que hacer referencia obligatoria al Proyecto Tuning 2 , en el cual se concreta todo lo expuesto anteriormente. Este documento recoge las competencias que han de alcanzar los universitarios y las clasifica en tres categorías: instrumentales, interpersonales y sistémicas (Jiménez, 2011).

\begin{tabular}{|l|l|l|}
\hline INSTRUMENTALES & INTERPERSONALES & SISTÉMICAS \\
\hline $\begin{array}{l}\text { Capacidad de análisis y síntesis; } \\
\text { conocimientos generales básicos; } \\
\text { comunicación oral y escrita en la } \\
\text { lengua propia; habilidades básicas } \\
\text { en la utilización del ordenador, etc. }\end{array}$ & $\begin{array}{l}\text { Trabajo en equipo, compromiso } \\
\text { ético. }\end{array}$ & $\begin{array}{l}\text { Capacidad de aplicar los conocimi- } \\
\text { entos en la práctica; capacidad de } \\
\text { aprender; habilidad para trabajar } \\
\text { de forma autónoma, etc. }\end{array}$ \\
\hline
\end{tabular}

Tabla 1. Clasificación de las competencias.

En esta línea de innovación, la formación debe llevarse a cabo, como bien dice Zabala (2009:64) "mediante un currículo centrado en las actividades profesionales de la docencia y el objeto de estudio del cual esté basado en buenas prácticas y en las soluciones a los problemas que la enseñanza plantea. En definitiva, una formación en la cual los conocimientos teóricos indispensables estén relacionados con las actividades reales de aula".

Por tanto, nuestro objetivo para la adquisición de la competencia literaria por parte de nuestro alumnado no vendrá condicionado exclusivamente por la adquisición de conocimientos, sino fundamentalmente por el desarrollo de habilidades, de procedimientos cognitivos y de actitudes que favorecerán el aprendizaje significativo, es decir un aprendizaje globalizador, así como el desarrollo de nuevas capacidades y destrezas. Un aprendizaje, por tanto, que primará los procesos sobre los resultados. Un aprendizaje que potencie la participación del alumno en la cons-trucción de su competencia literaria y favorezca el uso de una metodología participativa en la que se integren los procedimientos y las actividades que favorezcan los fines formativos de la recepción literaria, o sea, la educación para el goce estético (Mendoza, 2004).

2 Es un proyecto dirigido desde la esfera universitaria que tiene por objeto ofrecer un planteamiento concreto que posibilite la aplicación del proceso de Bolonia en el ámbito de las disciplinas o áreas de estudio y en el de las instituciones de educación superior. (Recuperado de http:// tuning.unideusto.org/tuningeu/). 
La poesía siempre ha sido la gran olvidada de las aulas como algunos autores aseveran, (Bordons, 2009; Cerrillo, 2007a), y en cambio, es el primer género literario con el que se entra en contacto en los primeros años de la vida, puesto que a partir de la poesía, los niños y niñas aprenden e interiorizan las primeras estructuras del lenguaje. Así, podemos apreciar que la tradición popular y la oralidad proporcionan al pequeño los primeros textos de su corta existencia: canciones de cuna, poemas, juegos lingüísticos, trabalenguas... El niño organiza el universo a través del juego y de la expresión poética, imaginativa y creadora (Huertas, 2007). Entra de lleno en el mundo del lenguaje a través de la poesía. La poesía no es solamente factor de su desarrollo personal, sino que también favorecerá la participación en la sociedad, el sentimiento de pertenecer a una colectividad y potenciará la solidaridad (Bordons, 2009), de aquí que tenga una gran importancia en un mundo donde la multiculturalidad y el multilingüismo están actualmente presentes en las aulas. Introducir la poesía en la escuela a edades tan tempranas nos proporcionará acercarla a los pequeños y enseñarles a apreciarla, incluso a amarla, para que puedan percibir que la poesía es algo útil porque hace ver las cosas de manera diferente, porque educa la sensibilidad y porque desarrolla el espíritu creativo (Cerrillo, 2007b). Consideramos que desarrollar la sensibilidad y el gusto por la estética a estas edades es un factor fundamental para crear buenos lectores de poesía en un futuro, visualizando el poema como una obra de arte, lo que convierte al futuro maestro en el catalizador del aprendizaje del niño en esta etapa. Hablaríamos aquí, evidentemente, de una motivación de la belleza y un aprendizaje de lo bello. Para acercar a los niños y niñas al lenguaje poético es interesante conocerlo, valorar los sentimientos que produce y memorizarlo, pero también es interesante saber producirlo (Fons, 2008).

Nuestro propósito con esta experiencia es introducir a nuestro alumnado en este maravilloso mundo, cautivar al futuro maestro a través de la poesía y sentar la base para su normalización en el aula proporcionándole los recursos necesarios. Partiendo de este objetivo común, pretendemos concretar una serie de estrategias que tendremos en cuenta para trabajar la poesía en el aula de infantil de 5 años. La valoración de los textos poéticos y el fomento del caudal creativo del lenguaje incluyen toda una serie de factores cognitivos, psicológicos y éticos que podemos encontrar en el aprendizaje de la poesía. Y este nuevo planteamiento y forma de aprender tendrá en cuenta los siguientes objetivos de aprendizaje de la competencia literaria, eso sí, desde la perspectiva conjunta del alumno-maestro:

\begin{tabular}{|l|}
\hline OBJETIVOS DE APRENDIZAJE \\
\hline Comprender una muestra de textos literarios diversos. \\
\hline Aprender cosas a través de la literatura. \\
\hline Contribuir a la socialización y a la estructuración del mundo del aprendiz a través de los textos literarios. \\
\hline Prepararlos para fomentar el gusto por la lectura. \\
\hline Fomentar su interés creativo. \\
\hline Configurar la personalidad literaria del niño. \\
\hline
\end{tabular}

Tabla 2. Objetivos de aprendizaje de la competencia literaria.

\section{Experiencia didáctica en el aula de Magisterio. Taller de poesía}

La experiencia de innovación educativa se llevó a cabo durante el curso académico 2016-2017 en el marco de la asignatura Recursos para la Educación Literaria. Esta asignatura es una optativa de 
6 ECTS que se ofrece en el cuarto curso de la titulación de Grado de Educación Infantil. Dicha experiencia se enmarca en el bloque temático 2 de la asignatura: «La poesía en el aula de infantil». Se trata de un taller de poesía que se realiza dentro de las sesiones de aula con asesoramiento y guía de la profesora con una temporalización de 4 horas a la semana durante los meses de febrero a mayo. La ponderación total del taller constituye el 50\% de la evaluación de la asignatura.

Proponemos este taller porque durante la etapa de Educación Infantil es cuando los más pequeños se inician en el aprendizaje de la lectoescritura y por tanto, los maestros y las maestras tienen que tener recursos y estrategias para planificar ese proceso de enseñanza-aprendizaje. Tanto la lectura como la escritura son procesos interpretativos a través de los cuales se construyen significados; eso implicará que leer y escribir son primordialmente actividades a través de las cuales construimos y ampliamos nuestro conocimiento del mundo que nos rodea (Díez, 2009).Y evidentemente, es un proceso en el que el alumnado tiene que participar, por ello consideramos que la lectoescritura se tiene que tratar a partir de actividades significativas para que los más pequeños vean el sentido y la necesidad de aprender a leer y escribir.

Por este motivo, planteamos a nuestro alumnado universitario la enseñanzaaprendizaje de la poesía como un recurso no solo para desarrollar la competencia literaria sino también como recurso lingüístico. Es un género que a los más pequeños les resulta ameno y próximo. La musicalidad que ofrecen los versos hace que les sea fácil de memorizar y al mismo tiempo, la rima constituye una buena estrategia para hacerles jugar con la lengua. Con esta elección pretendíamos que nuestro alumnado desarrollara no solo las competencias específicas de la asignatura, sino también las competencias genéricas del Grado.

Para impartir la asignatura elegimos la metodología de rincones. Por una parte, porque te-níamos en mente conceptos tales como, aprendizaje significativo, aprendizaje autónomo y conexión con la realidad. Por otra parte, porque es un método de trabajo implementado en Educación Infantil y teniendo en cuenta que nuestro alumnado estaba estudiando esta rama de Magisterio, consideramos que era una buena manera de ponerse en contacto con esta metodología activa-participativa ya que los rincones proporcionan una serie de ventajas de orden psicopedagógico, tan importantes en estas edades, como las propuestas por Trueba (1999) para el desarrollo evolutivo del niño:

-El rincón posibilita educar al pequeño desde la base de la colectividad y proporciona un modelo basado en la socialización.

-Favorece y fomenta la autonomía personal, tan importante en estas etapas.

-Fomenta de modo natural los hábitos del orden.

-Facilita el "aprender jugando".

-Estimula la investigación y la curiosidad.

-Y en este caso en concreto, contribuye a aprender poesía inventando.

Una vez realizada la investigación sobre la metodología de rincones, los alumnos pasaron a diseñar el "Rincón de la poesía" en el cual tenían que trabajar diferentes subgéneros poéticos y planificar actividades dirigidas a alumnado de segundo ciclo de Educación Infantil. Esto les permitió planificar, estructurar, crear, dialogar, etc.; en definitiva, llevar a la práctica acciones significativas para su aprendizaje, teniendo en cuenta que el planteamiento del trabajo por rincones implica la necesidad de establecer estrategias organizativas a fin de responder a los diferentes in- 
tereses y ritmos de aprendizaje de cada niño o niña (Fernández, Quer y Securún, 2009). De esta manera, nuestros discentes pudieron poner en práctica diversas actividades relacionadas con la poesía a partir de distintos grados de dificultad e implicación.

Pasamos a describir a continuación los objetivos y la secuencia didáctica llevada a cabo en nuestra experiencia:

\section{OBJETIVOS}

O2 Conocer y valorar los enfoques y los procesos para la enseñanza y el aprendizaje de la expresión y la comprensión oral y escrita.

O4 Diseñar, planificar y evaluar procesos de enseñanza y aprendizaje de forma individual y en colaboración con otros docentes y profesionales del centro.

O6 Comprender que la observación sistemática es un instrumento básico para poder reflexionar sobre la práctica y la realidad y también contribuir a la innovación y a la mejora en la educación.

O9 Reflexionar sobre las prácticas de aula para innovar y mejorar la tarea docente.

Tabla 3. Objetivos extraídos de la guía docente de la asignatura.

\section{SECUENCIA DIDÁCTICA}

Formación de grupos de trabajo y explicación de la tarea.

Presentación de la poesía como recurso en el aula y posterior investigación de diferentes subgéneros poéticos.

(Acceso al conocimiento y planificación).

Elección de un subgénero poético para la realización del rincón.

Pautas para el diseño de actividades y metodología a seguir.
En primer lugar, se dividió al grupo-clase en grupos de 4 componentes y se explicó la tarea que se iba a llevar a cabo mediante el taller de poesía y su posterior implementación.

Se explicó al alumnado la importancia del recurso de la poesía como género para trabajar en las aulas, y se presentaron los subgéneros poéticos que se iban a trabajar, concretamente: el caligrama, el haiku, el acróstico, las adivinanzas y el poema ilustrado. Seleccionamos dichos subgéneros porque dentro de la poesía consideramos que eran los más adecuados para la etapa de infantil por su sencillez y por su aportación visual. Una vez realizada esta explicación, el alumnado tenía que hacer una pequeña investigación sobre cada uno de estos subgéneros para conocer e identificar sus características, pasando posteriormente a una puesta en común en el grupo-aula.

Después de la puesta en común, cuando ya habían asimilado las características de los subgéneros, se les explicó que el trabajo consistía en crear entre todo el grupo-clase un rincón de poesía para un aula de infantil, constituido por los diferentes subgéneros poéticos estudiados. A cada grupo se le asignó un subgénero y tenían que diseñar diversas actividades para trabajarlo con alumnado del tercer curso de infantil de un colegio de la zona, con el que previamente se había contactado para conocer las características del alumnado.

Se les explicaron las estrategias básicas a partir de las cuales se puede trabajar la poesía en el aula de infantil para que tuvieran un punto de referencia a la hora de planificar y estructurar las actividades, como por ejemplo recuperar el repertorio de poemas de autores que ya han sido recopilados, memorizarlos y jugar con ellos; hacerles gozar de las posibilidades expresivas, sonoras y sensibles de la palabra; propiciar con la poesía una serie de experiencias positivas para que los niños aprendan a expresar sus sentimientos, etc.

Para poder llevar a cabo la realización del rincón de poesía, se les explicó la metodología de rincones así como las pautas a seguir. 


\begin{tabular}{|l|l|}
\hline \multirow{2}{*}{$\begin{array}{l}\text { Diseño de actividades para tra- } \\
\text { bajar el subgénero. }\end{array}$} & $\begin{array}{l}\text { Una vez claras las premisas de dicha metodología y las pautas para el di- } \\
\text { seño de las actividades, cada grupo tenía que elaborar una ficha de trabajo } \\
\text { en la cual se recogieran los puntos siguientes: justificación del trabajo, ob- } \\
\text { jetivos (según el currículo) y descripción de las actividades, como guía para } \\
\text { el desarrollo del trabajo. Para el diseño de las actividades se les indicó que } \\
\text { no asumieran que todos los alumnos estaban en el mismo nivel de lectoes- } \\
\text { critura, sino que se tenía que respetar el ritmo de aprendizaje de cada uno. } \\
\text { Por tanto, estas actividades tenían que ir en gradación de dificultad, pero } \\
\text { también se tenían que poder ajustar a los diferentes estadios de escritura, } \\
\text { como son la fase pre-silábica, la escritura espontánea o el caso de aquellos } \\
\text { niños que ya saben escribir (Díez, 2009). }\end{array}$ \\
\hline $\begin{array}{l}\text { Exposición de las actividades en } \\
\text { el aula. }\end{array}$ & $\begin{array}{l}\text { Una vez terminados los rincones se expusieron en el aula para reflexionar } \\
\text { sobre el tipo de actividades que se habían diseñado. Se realizó una coeva- } \\
\text { luación mediante una rúbrica (Tabla 5). }\end{array}$ \\
\hline $\begin{array}{l}\text { Implementación con alumnado } \\
\text { de } 5 \text { años de Educación Infantil. }\end{array}$ & $\begin{array}{l}\text { Se eligió un día y una hora acordada con el colegio para poner en práctica } \\
\text { la experiencia. Nuestros discentes se distribuyeron en los espacios libres } \\
\text { de la facultad realizaron el montaje de los diferentes rincones que compo- } \\
\text { nían el “Rincón de poesía". Se organizó al alumnado de infantil en pequeños } \\
\text { grupos para que todos pudieran pasar por los diferentes espacios creados. }\end{array}$ \\
\hline $\begin{array}{l}\text { Reflexión y valoración de la im- } \\
\text { plementación. }\end{array}$ & $\begin{array}{l}\text { En la siguiente sesión de aula se realizó una puesta en común con el grupo } \\
\text { clase de Magisterio para valorar la experiencia llevada a cabo. }\end{array}$ \\
\hline
\end{tabular}

Tabla 4. Explicación de la secuencia didáctica.

\subsection{Evaluación}

Para terminar de describir esta experiencia educativa haremos referencia a la evaluación. Si queremos que nuestro alumnado adquiera competencias y, en consecuencia, implementamos metodologías centradas en el aprendizaje para conseguir nuestro objetivo, la evaluación tendrá que reflejar la consecución de dichas competencias. Como ya hemos dicho, por competencia se entiende la capacidad de actuar en situaciones complejas e imprevisibles aplicando los conocimientos, procedimientos y actitudes aprendidas; por tanto, la evaluación tendrá que posibilitar que se pueda comprobar que realmente ha sido así, puesto que nos enfrentamos a un conjunto integrado y complejo de saberes de distintos tipos. Siguiendo a Sanmartí (2009), la evaluación tendrá que ser diferente de las actividades de evaluación tradicionales, puesto que están más orientadas a comprobar si el alumnado es capaz de reproducir el conocimiento enseñado. Esta idea enlaza con lo que conocemos como el alineamiento constructivo de Biggs (s.f.), en el que dicho autor presenta el proceso de enseñanza-aprendizaje como un ecosistema, es decir, como un sistema equilibrado en el que todos los componentes se apoyan los unos con los otros y si uno de ellos falla, deriva en un aprendizaje superficial. En nuestra experiencia, mediante la creación de los rincones, el alumnado ha activado todo el conocimiento adquirido sobre los géneros poéticos y además, ha puesto en funcionamiento toda una serie de habilidades y capacidades para elaborar en primer lugar, un plan de trabajo sobre qué querían que los alumnos de infantil aprendieran, y plantear en segundo lugar, toda una serie de actividades para que el proceso de enseñanza-aprendizaje fuera satisfactorio, que era otro de nuestros objetivos.

Por otra parte, el hecho de utilizar una rúbrica para evaluar los rincones ha sido muy eficaz, tanto a nivel docente como del alumnado, porque tal y como explica Sanmartí (2009), normalmente 
los criterios de evaluación son implícitos, y los discentes los tienen que intuir. Algunos alumnos acostumbran a reconocer qué es lo que se valorará de su trabajo, pero la mayoría no ha desarrollado esa capacidad. Sin identificar los criterios de evaluación es muy difícil realizar bien una tarea. Así pues, mediante esta rúbrica, el alumnado ha tenido presente en todo momento qué se le exigía en la elaboración de los rincones. Presentamos a continuación la rúbrica que hemos usado:

\begin{tabular}{|c|c|c|c|c|}
\hline & Sobresaliente & Notable & Aprobado & Suspenso \\
\hline $\begin{array}{l}\text { Materiales } \\
\text { Capacidad y orden }\end{array}$ & $\begin{array}{l}\text { Hay materiales } \\
\text { suficientes } \\
\text { para que los } \\
\text { alumnos trabajen } \\
\text { cómodamente y } \\
\text { simultáneamente. } \\
\text { Además todos los } \\
\text { materiales están } \\
\text { bien organizados } \\
\text { y clasificados } \\
\text { con carpetas } \\
\text { o elementos } \\
\text { similares para que } \\
\text { se mantengan } \\
\text { ordenados. }\end{array}$ & $\begin{array}{l}\text { Hay materiales } \\
\text { suficientes } \\
\text { para que los } \\
\text { alumnos trabajen } \\
\text { cómodamente y } \\
\text { simultáneamente } \\
\text { y casi todos los } \\
\text { materiales están } \\
\text { organizados y } \\
\text { clasificados. }\end{array}$ & $\begin{array}{l}\text { Hay materiales } \\
\text { suficientes, pero } \\
\text { no están del todo } \\
\text { bien organizados, } \\
\text { lo cual genera } \\
\text { una sensación de } \\
\text { desorden. }\end{array}$ & $\begin{array}{l}\text { No hay materiales } \\
\text { para que los } \\
\text { alumnos trabajen } \\
\text { cómodamente y } \\
\text { simultáneamente } \\
\text { y están } \\
\text { desordenados, con } \\
\text { lo cual es difícil } \\
\text { encontrarlos en el } \\
\text { rincón. }\end{array}$ \\
\hline $\begin{array}{l}\text { Materiales } \\
\text { Claridad y } \\
\text { significatividad } \\
\text { del contenido del } \\
\text { material }\end{array}$ & $\begin{array}{l}\text { Los materiales } \\
\text { son claros y } \\
\text { lógicamente } \\
\text { significativos. } \\
\text { Consiguen explicar } \\
\text { en qué consiste } \\
\text { el género poético. } \\
\text { Están presentes } \\
\text { todos los aspectos } \\
\text { indicados, lo } \\
\text { cual facilita el } \\
\text { aprendizaje. }\end{array}$ & $\begin{array}{l}\text { Los materiales } \\
\text { son claros, pero } \\
\text { no están bien } \\
\text { secuenciados. } \\
\text { Consiguen explicar } \\
\text { en qué consiste } \\
\text { el género poético, } \\
\text { pero no está } \\
\text { presente alguno } \\
\text { de los elementos } \\
\text { indicados. }\end{array}$ & $\begin{array}{l}\text { Algunos materiales } \\
\text { son claros y } \\
\text { otros no tanto. } \\
\text { Consiguen explicar } \\
\text { en qué consiste } \\
\text { el género poético, } \\
\text { pero no están } \\
\text { presentes algunos } \\
\text { de los elementos } \\
\text { indicados. }\end{array}$ & $\begin{array}{l}\text { Los materiales } \\
\text { no son claros } \\
\text { ni lógicamente } \\
\text { significativos. No } \\
\text { consiguen explicar } \\
\text { bien en qué } \\
\text { consiste el género } \\
\text { poético. }\end{array}$ \\
\hline $\begin{array}{l}\text { Materiales } \\
\text { Diseño y } \\
\text { originalidad }\end{array}$ & $\begin{array}{l}\text { Los materiales } \\
\text { tienen un diseño } \\
\text { cuidado y } \\
\text { original. Facilita la } \\
\text { comprensión del } \\
\text { contenido. }\end{array}$ & $\begin{array}{l}\text { Los materiales } \\
\text { tienen un } \\
\text { diseño cuidado } \\
\text { que facilita la } \\
\text { comprensión. Pero } \\
\text { el medio elegido } \\
\text { no aporta calidad a } \\
\text { la presentación del } \\
\text { material. }\end{array}$ & $\begin{array}{l}\text { Los materiales } \\
\text { tienen un diseño } \\
\text { simple y poco } \\
\text { original que } \\
\text { no ayuda a la } \\
\text { comprensión } \\
\text { del contenido. El } \\
\text { medio elegido no } \\
\text { es muy adecuado. }\end{array}$ & $\begin{array}{l}\text { Los materiales } \\
\text { tienen un diseño } \\
\text { deficiente y } \\
\text { poco original } \\
\text { que dificulta la } \\
\text { comprensión } \\
\text { del contenido. El } \\
\text { medio elegido no } \\
\text { es adecuado. }\end{array}$ \\
\hline Actividades & $\begin{array}{l}\text { Las actividades } \\
\text { son adecuadas } \\
\text { al nivel de los } \\
\text { destinatarios. Se } \\
\text { ajustan al tiempo } \\
\text { establecido y } \\
\text { consiguen que } \\
\text { quien las realice } \\
\text { adquieran una idea } \\
\text { bastante amplia de } \\
\text { lo que es el género } \\
\text { poético. }\end{array}$ & $\begin{array}{l}\text { Las actividades } \\
\text { son adecuadas } \\
\text { al nivel de los } \\
\text { destinatarios. } \\
\text { Consiguen que } \\
\text { quien las realice } \\
\text { aprenda lo esencial } \\
\text { del género poético, } \\
\text { pero no permiten } \\
\text { hacerse una idea } \\
\text { completa. }\end{array}$ & $\begin{array}{l}\text { Las actividades } \\
\text { son adecuadas } \\
\text { al nivel de los } \\
\text { destinatarios. Pero } \\
\text { no consiguen que } \\
\text { quien las realice } \\
\text { aprenda lo esencial } \\
\text { del género poético. }\end{array}$ & $\begin{array}{l}\text { Las actividades } \\
\text { son inadecuadas } \\
\text { al nivel de los } \\
\text { destinatarios. No } \\
\text { consiguen que } \\
\text { quien las realice } \\
\text { aprenda lo esencial } \\
\text { del género poético. }\end{array}$ \\
\hline
\end{tabular}

Tabla 5. Rúbrica de evaluación del trabajo por rincones.

Para finalizar presentamos a continuación una tabla con la información pertinente sobre los criterios de evaluación, las competencias, los resultados de aprendizaje, los instrumentos y las evidencias de evaluación de la experiencia. 


\begin{tabular}{|c|c|c|c|c|}
\hline $\begin{array}{l}\text { Criterios de evalu- } \\
\text { ación }\end{array}$ & Competencias & $\begin{array}{l}\text { Resultados de } \\
\text { aprendizaje }\end{array}$ & $\begin{array}{l}\text { Instrumentos de } \\
\text { evaluación }\end{array}$ & $\begin{array}{l}\text { Evidencias de eva- } \\
\text { luación }\end{array}$ \\
\hline $\begin{array}{l}\text { Ha expuesto de } \\
\text { forma correcta y } \\
\text { fluida las activi- } \\
\text { dades diseñadas } \\
\text { utilizando un re- } \\
\text { gistro adecuado a } \\
\text { la situación comu- } \\
\text { nicativa. }\end{array}$ & $\begin{array}{l}\text { E48 Comprender } \\
\text { el paso de la ora- } \\
\text { lidad a la escritura } \\
\text { y conocer los dife- } \\
\text { rentes registros y } \\
\text { usos de la lengua. }\end{array}$ & $\begin{array}{l}\text { R1 El alumnado } \\
\text { conoce y domi- } \\
\text { na técnicas de } \\
\text { expresión oral y } \\
\text { escrita para favo- } \\
\text { recer el desarrollo } \\
\text { de las habilidades } \\
\text { lingüísticas. }\end{array}$ & $\begin{array}{l}\text { Exposiciones orales } \\
\text { de las actividades } \\
\text { creadas para el } \\
\text { "Rincón de poesía" } \\
(30 \%)\end{array}$ & $\begin{array}{l}\text { Registro de la prue- } \\
\text { ba oral. }\end{array}$ \\
\hline $\begin{array}{l}\text { Ha reflexionado } \\
\text { sobre la metodo- } \\
\text { logía de rincones } \\
\text { y ha aportado } \\
\text { información sobre } \\
\text { los subgéneros } \\
\text { poéticos. }\end{array}$ & $\begin{array}{l}\text { CG9 Reflexionar } \\
\text { sobre la práctica } \\
\text { de aula para inno- } \\
\text { var y mejorar la } \\
\text { tarea docente. } \\
\text { E52 Conocer y } \\
\text { utilizar adecuada- } \\
\text { mente recursos } \\
\text { para la animación } \\
\text { a la lectura y la } \\
\text { escritura. }\end{array}$ & $\begin{array}{l}\text { R4 El alumnado } \\
\text { conoce fuentes } \\
\text { básicas de la in- } \\
\text { vestigación en el } \\
\text { campo de la Didác- } \\
\text { tica de la lengua y } \\
\text { la literatura, como } \\
\text { la observación y el } \\
\text { análisis de la acti- } \\
\text { vidad en el aula e } \\
\text { identifica el objeto } \\
\text { y la metodología } \\
\text { utilizada. }\end{array}$ & $\begin{array}{l}\text { Ficha de trabajo } \\
\text { grupal del diseño } \\
\text { de actividades para } \\
\text { trabajar el subgéne- } \\
\text { ro }(20 \%)\end{array}$ & $\begin{array}{l}\text { Documento guía de } \\
\text { actividades. }\end{array}$ \\
\hline $\begin{array}{l}\text { Ha diseñado acti- } \\
\text { vidades adecuadas } \\
\text { para favorecer el } \\
\text { aprendizaje del } \\
\text { subgénero poéti- } \\
\text { co. } \\
\text { Ha diseñado acti- } \\
\text { vidades adecuadas } \\
\text { para la etapa edu- } \\
\text { cativa a la que van } \\
\text { dirigidas. }\end{array}$ & $\begin{array}{l}\text { CG4 Conocer e } \\
\text { integrar los obje- } \\
\text { tivos, contenidos } \\
\text { curriculares y crite- } \\
\text { rios de evaluación } \\
\text { de la Educación } \\
\text { Infantil. Diseñar, } \\
\text { planificar y evaluar } \\
\text { proyectos de en- } \\
\text { señanza. } \\
\text { CG22 Ser capaz } \\
\text { de justificar las } \\
\text { decisiones relati- } \\
\text { vas al aprendizaje, } \\
\text { la evaluación y } \\
\text { la educación del } \\
\text { alumnado y ser } \\
\text { consecuente con } \\
\text { la toma de decisi- } \\
\text { ones. } \\
\text { E58 Elaborar pro- } \\
\text { puestas didácticas } \\
\text { que fomenten la } \\
\text { percepción y la } \\
\text { expresión, las ha- } \\
\text { bilidades motrices } \\
\text { y la creatividad. }\end{array}$ & $\begin{array}{l}\text { R5 El alumnado } \\
\text { manipula recursos } \\
\text { para la educación } \\
\text { literaria que posi- } \\
\text { biliten su eficacia } \\
\text { comunicativa. }\end{array}$ & $\begin{array}{l}\text { Diseño final del } \\
\text { "Rincón de poesía" } \\
\text { con el subgénero } \\
\text { asignado }(30 \%) \\
\text { Prueba final teórica } \\
(20 \%)\end{array}$ & $\begin{array}{l}\text { Fotografías y rúbri- } \\
\text { ca de evaluación. } \\
\text { Examen escrito. }\end{array}$ \\
\hline
\end{tabular}

Tabla 6. Cuadro de criterios de evaluación, competencias, resultados de aprendizaje, instrumentos y evidencias de evaluación.

\section{Conclusiones}

Después de la experiencia llevada a cabo y tomando como referencia las evidencias de evaluación, hemos podido constatar que los grupos de alumnos universitarios con los que se ha implementado, han reconocido que con esta metodología de trabajo socio constructivo, han aprendido más y 
mejor, lo que les ha posibilitado la adquisición de la competencia de «aprender a aprender». Hecho que se puede corroborar con los buenos resultados obtenidos en la asignatura. Partiendo de la puesta en común reflexiva una vez realizada la experiencia con el alumnado de infantil, podemos afirmar que esta metodología les ha despertado ilusión y ganas de trabajar, puesto que la tarea final era un producto atractivo y enlazaba directamente con su futura realidad profesional. Con este recurso podemos confirmar que el alumnado ha ganado en autonomía personal y en toma de decisiones y se les ha preparado para su incorporación en el aula, destacando así la adquisición de la competencia «aprender a hacer».

Igualmente los futuros docentes al implementar los rincones con alumnado de infantil han podido observar, de forma directa y sistemática, cómo el aprendizaje de la lectoescritura con esta metodología, propicia la participación del alumnado, facilita la diversificación c urricular, t iene en cuenta la motivación y sus intereses, y plantea actividades de lectura y escritura con sentido, facilitando la interacción y el trabajo cooperativo. Así mismo, a través de las actividades generadas han sido capaces de destacar la belleza de la palabra, el trabajo de los sentimientos, el desarrollo de la sensibilidad y del sentido estético contribuyendo así a la adquisición de la competencia «aprender a ser». Todos estos aspectos los reforzaron las dos tutoras de los grupos participantes en sus propias sesiones de aula, a través de la creación de rimas, pareados, pictogramas poéticos, etc., referentes a lo experimentado en el «Rincón de poesía».

En definitiva, c on e sta e xperiencia p odemos c oncluir q ue h emos conseguido que n uestro alumnado universitario adquiera una enseñanza basada en las competencias instrumentales, interpersonales y sistémicas que se exigen desde el Espacio Europeo de Educación Superior. Así como las competencias genéricas, específicas y transversales de la materia Recursos para la Educación Literaria, puesto que han sido capaces de ampliar su formación literaria con la adquisición de conocimientos sobre los géneros poéticos trabajados; de diseñar actividades siguiendo el currículo de infantil e implementar el "Rincón de poesía". En conclusión se ha conseguido un aprendizaje significativo.

\section{Referencias bibliográficas}

Biggs, J. (s.d.). Constructive alignment in university teaching. HERDSA Review of Higher Education, 1, 5-22.

Bordons, G. (coord.) (2009). Poesia i educació. D’internet a l'aula. Biblioteca d'Articles. Sèrie Didàctica de la Llengua i de la Literatura, 170.

Cerrillo, P.C. (2007a). Literatura infantil y juvenil y educación literaria. Barcelona: Octaedro.

Cerrillo, P.C. (2007b). Poesía y escuela: reivindicación de la Poesía Infantil. Lenguaje y Textos, $26,33-52$.

Díez de Ulzurrun Pausa, A. (coord.) (2009). L'aprenentatge de la lectoescriptura des d’una perspectiva constructivista. Vol. I; Vol. II. Barcelona: Graó.

Fernández, E., Quer, L. \& Securún, R. M. (2009). Rincón a rincón. Barcelona: Octaedro-Rosa Sensat. Fons Esteve, M. (2008). Llegir i escriure per viure. Barcelona: La Galera.

Huertas Gómez, R. (2007). Poesía popular infantil y creatividad. Madrid: CCS. 
Jiménez, M.A. (2011). Cómo diseñar y desarrollar el currículo por competencias. Madrid: PPCEditorial.

Mendoza Fillola, A. (2004). La educación literaria. Bases para la formación de la competencia lecto-literaria. Málaga: Aljibe.

Sanmartí, N. (2009). Avaluar per desenvolupar competències. Avaluar competències. Guix, 359, 49-53.

Trueba Marcano, B. (1999). Talleres integrales en Educación Infantil. Madrid: Ediciones de la Torre.

Tuning. (2014). Una introducción a Tuning Educational Structures in Europe. La contribución de las universidades al proceso de Boloña. Recuperado de www.unideusto.org.

Zabala Vidiella, A. (1995). Projectes, projectes de treball, projectes de recerca del medi. Revisió històrica i validesa actual. Articles de Didàctica de la Llengua i de la Literatura, 3.

Zabala Vidiella, A. (2009). L'ensenyament basat en competències, una nova oportunitat. Guix, 351, 63-66. 